\title{
SYSTEMS OF PARTIAL DIFFERENTIAL OPERATORS WITH FUNDAMENTAL SOLUTIONS SUPPORTED BY A CONE
}

\author{
KIRK E. LANCASTER AND BENT E. PETERSEN ${ }^{1}$
}

\begin{abstract}
Necessary and sufficient conditions are given for a system of partial differential operators to have a fundamental solution supported by a convex salient cone. As a simple application an overdetermined Cauchy problem is solved.
\end{abstract}

If $A$ is a subset of $\mathbf{R}^{n}$ and $\mathscr{F}$ is a space of distributions on $\mathbf{R}^{n}$ we denote by $\mathscr{F}_{A}$ the space of distributions in $\mathscr{F}$ which have supports contained in $A$. We denote by $\mathscr{D}^{\prime}$ the space of all distributions on $\mathbf{R}^{n}$, by $\mathcal{S}^{\prime}$ the space of temperate distributions, and by $\mathcal{E}$ the space of infinitely differentiable functions on $\mathbf{R}^{n}$. If $\Gamma$ is a closed convex cone in $R^{n}$ with vertex at the origin, we denote by $\Gamma^{+}$the dual cone defined by $\Gamma^{+}=\left\{\xi \in R^{n} \mid\langle\xi, x\rangle \geqslant 0, x \in \Gamma\right\}$. Then $\Gamma^{++}=\Gamma$. The interior $\Gamma_{0}^{+}$of $\Gamma^{+}$is nonempty if and only if $\Gamma$ is salient, i.e. contains no subspace other than $\{0\}$. If $\Gamma$ is salient then $\mathscr{D}_{\Gamma}^{\prime}$ is a commutative ring relative to convolution. If $H$ is a closed half-space with interior normal $\eta \in \Gamma_{0}^{+}$then $\mathscr{D}_{H}^{\prime}$ is a $\mathscr{D}_{\Gamma}^{\prime}$-module, and differentiation commutes with convolution in the usual fashion. Finally we note $\delta_{\Gamma}^{\prime}$ is a subring of $\mathscr{D}_{\Gamma}^{\prime}$. This fact is proved in the appendix below.

Let $P(z)$ be a $p \times q$ matrix over $C\left[z_{1}, \ldots, z_{n}\right]$ and denote by $P(D)$ the system of partial differential operators obtained by replacing $z_{j}$ in $P(z)$ by $\partial / \partial x_{j}$. If $p<q$ then a fundamental solution for $P(D)$ is a $q \times p$ matrix $K$ over $\mathscr{D}^{\prime}$ such that

$$
P(D) K=\delta I
$$

where $I$ is the $p \times p$ identity matrix and $\delta$ is the Dirac measure at 0 . In case $p=q$ then $P(D)$ has a fundamental solution with support in the closed convex salient cone $\Gamma$ if and only if $P(D)$ is hyperbolic with respect to each direction in $\Gamma_{0}^{+}$, [1]. In case $p=q=1$ then $P(D)$ has a temperate fundamental solution with support in the closed convex salient cone $\Gamma$ if and only if $P(z) \neq 0$ for each $z$ in $\Gamma_{0}^{+}+i R^{n}$. This fact may be proved by means of an elementary inequality for polynomials, as is done in the introduction to [9]. The temperate case with $p=1, q>1$ is also considered in ([8], [9]) and may easily be generalized as is done below. In this note we will give a sufficient, and in case $\Gamma$ is semialgebraic, necessary condition for $P(D)$ in the case $p \leqslant q$ to have a fundamental solution $K$ with support in the closed convex salient cone $\Gamma$. Our methods do not apply in the nonsalient case. The scalar case $p=q=1$ with $\Gamma$ nonsalient has been considered by A. Enqvist in [3] and in the temperate case in [4]. We will prove the following two theorems.

Received by the editors January 30, 1979.

AMS (MOS) subject classifications (1970). Primary 35E05; Secondary 35 L55.

${ }^{1}$ Research supported in part by NSF MCS 74-06803-A03. 
Theorem 1. Let $\Gamma$ be a closed convex salient cone and $p<q$. Then $P(D)$ admits a temperate fundamental solution $K$ with support in $\Gamma$ if and only if for each $z$ in the tube $\Gamma_{0}^{+}+i R^{n}$ the matrix $P(z)$ has rank $p$.

TheOREM 2. Let $\Gamma$ be a closed convex salient cone and $p<q$. Then $P(D)$ admits a fundamental solution $K$ with support in $\Gamma$ if and, in the case $\Gamma$ is semialgebraic, only if there exists a convex open set $U$ in $\Gamma_{0}^{+}$such that $t U \subseteq U$ for $t>1, \Gamma_{0}^{+}=U t U$ $(t>0)$ and for each $z$ in the tube $U+i R^{n}$ the matrix $P(z)$ has rank $p$.

In the case $p=q$ we may dispense with the hypothesis that $\Gamma$ is semialgebraic. There are at least two ways to do this. If $P(D)$ has a fundamental solution with support in $\Gamma$ then the determinant det $P(D)$ is hyperbolic with respect to each direction in $\Gamma_{0}^{+}$. From the theory of scalar hyperbolic operators [5] it follows that det $P(D)$ is hyperbolic with respect to each direction in an open convex semialgebraic cone which contains $\Gamma_{0}^{+}$. By the lemma below we then obtain a closed convex semialgebraic cone $\Gamma^{\prime} \subseteq \Gamma$ such that $P(D)$ has a fundamental solution with support in $\Gamma^{\prime}$. Alternately, if $\Gamma$ is not assumed semialgebraic a modification of the proof of necessity produces an open set $U$ with the required properties other than convexity. In the case $p=q, \mathrm{~S}$. Bochner's theorem on tubes [6, Theorem 2.5.10] then shows we may replace $U$ by its convex hull.

We first reduce the $p \times q$ system to a $1 \times N$ system, $N=\left(\begin{array}{l}q \\ p\end{array}\right)$. The notation $|J|=p$ will mean that $J=\left(j_{1}, \ldots, j_{p}\right)$ where the $j_{k}$ are integers and $1<j_{k}<q$ for each $k$. For each such $J$ let $P^{J}(z)$ be the $p \times p$ matrix whose $k$ th column is the $j_{k}$ th column of $P(z)$ and let $Q_{J}(z)$ be the determinant of $P^{J}(z)$.

Lemma. Let $\Gamma$ be a closed convex salient cone and $p<q$. Then $P(D)$ admits a fundamental solution (respectively, a temperate fundamental solution) with support in $\Gamma$ if and only if there exist distributions (respectively, temperate distributions) $L_{J}$, $|J|=p$, with supports in $\Gamma$ such that

$$
\sum_{|J|=p}^{\prime} Q_{J}(D) L_{J}=\delta
$$

Here the prime over the summation symbol indicates that we sum only over $p$-indices $J=\left(j_{1}, \ldots, j_{p}\right)$ with $1<j_{1}<\cdots<j_{p} \leqslant q$. For the proof, suppose first that (2) holds with supp $L_{J} \subseteq \Gamma$. Let $Q_{J}^{i k}(z)$ be the $(i, k)$-cofactor of $P^{J}(z)$, that is $(-1)^{i+k}$ times the determinant of the matrix obtained from $P^{J}(z)$ by removing the $i$ th row and the $k$ th column. Then

$$
\sum_{h=1}^{p} P_{i j_{h}}(z) Q_{J}^{l h}(z)= \begin{cases}Q_{J}(z) & \text { if } l=i \\ 0 & \text { if } l \neq i\end{cases}
$$

where $J=\left(j_{1}, \ldots, j_{p}\right)$. If we set

$$
K_{j l}=\sum_{h=1}^{p} \sum^{\prime} Q_{J}^{l h}(D) L_{J}, \quad 1<j<q, 1<l<p,
$$

where the inner sum is over $|J|=p$ such that $j_{h}=j$, then supp $K_{j l} \subseteq \Gamma$ and

$$
\sum_{j=1}^{q} P_{i j}(D) K_{j l}=\sum_{|J|=p}^{\prime} \sum_{h=1}^{p} P_{i j h}(D) Q_{J}^{l h}(D) L_{J}
$$


whence (1) follows. If the $L_{J}$ are temperate, then so also are the $K_{j l}$.

Conversely suppose (1) holds with supp $K_{j l} \subseteq \Gamma$. Let $A_{i j}=P_{i j}(D) \delta$ so $A * K=$ $\delta I$. Since the distributions with supports in $\Gamma$ form a commutative ring with respect to convolution it makes sense to take the determinant. From the Binet-Cauchy formula we obtain

$$
\delta=\operatorname{det}(A * K)=\sum_{|J|=p}^{\prime}\left(\operatorname{det} A_{J}\right) *\left(\operatorname{det} K_{J}\right)
$$

where $A_{J}$ is the matrix whose $k$ th column is the $j_{k}$ th column of $A$ and $K_{J}$ is the matrix whose $k$ th row is the $j_{k}$ th row of $K$. Since $A_{J}=P^{J}(D)(\delta I)$ we see that $\operatorname{det} A_{J}=Q_{J}(D) \delta$. If we set $L_{J}=\operatorname{det} K_{J}$ then (2) follows and $\operatorname{supp} L_{J} \subseteq \Gamma$. If $K$ is temperate then the $L_{J}$ are temperate (see Appendix). Note it is not difficult to see if we start with $K$ and set $L_{J}=\operatorname{det} K_{J}$ then the construction at the beginning of the proof yields the original $K$.

The lemma is now proved and moreover Theorem 1 follows from the $p=1$ case which is considered in [8], [9]. The proof of the lemma is quite standard. The argument for example is similar to the argument in the $p=q$ case given in [1, Lemma 3.2]. The sufficiency of (2) in the $p<q$ case is the same as the argument in [11, Theorem 4.1]. We gave the argument, however, because prior to proceeding to the proof of Theorem 2 we will use the notation and proof of the lemma to solve an overdetermined Cauchy problem for a half-space when compatibility conditions are satisfied. Let $P^{t}(z)$ denote the transpose of the matrix $P(z)$.

Theorem 3. Let $\Gamma$ be a closed convex salient cone and $p<q$. Assume (1) holds with supp $K \subseteq \Gamma$. Let $\eta \in \Gamma_{0}^{+}$and let $H$ be the closed half-space $\left\{x \in R^{n}|\langle x, \eta\rangle\rangle\right.$ 0\}. If $w \in\left(\mathscr{D}^{\prime}\right)^{p}$ and if $\operatorname{supp}\left(P^{t}(D) w\right) \subseteq H$ then there exists a unique $u \in\left(\mathscr{D}^{\prime}\right)^{p}$ such that

$$
\text { supp } u \subseteq H, \quad P^{t}(D) u=P^{t}(D) w .
$$

Moreover, if $w \in \mathcal{E}^{p}$ then $u \in \mathcal{E}^{p}$.

We prove uniqueness first. Suppose $u \in\left(\mathscr{D}_{H}^{\prime}\right)^{p}$ and let $v=K^{t} * P^{t}(D) u$. Since $K_{j k} \in \mathscr{D}_{\Gamma}^{\prime}$ we have

$$
\begin{aligned}
v_{k} & =\sum_{j} K_{j k} * \sum_{h} P_{h j}(D) u_{h} \\
& =\sum_{j, h} P_{h j}(D) K_{j k} * u_{h}=u_{k}
\end{aligned}
$$

Thus $u=K^{t} * P^{t}(D) u$ for any $u \in\left(\mathscr{D}_{H}^{\prime}\right)^{p}$ which gives the uniqueness.

For existence we define $u \in\left(\mathscr{D}_{H}^{\prime}\right)^{p}$ by $u=K^{t} * P^{t}(D) w$. Note if $w$ is smooth, then so is $u$ which gives the last part. To see that $u$ is a solution, since we have no control over supp $w$ some care is required in commuting convolutions and differentiations. By the proof of the lemma we have $L_{J} \in \mathscr{D}_{\Gamma}^{\prime}$ such that

$$
K_{j k}=\sum_{h=1}^{p} \Sigma^{\prime} Q_{J}^{k h}(D) L_{J}
$$


where the inner sum is over $|J|=p$ with $j_{h}=j$. Then, since supp $L_{J} \subseteq \Gamma$,

$$
\begin{aligned}
u_{k} & =\sum_{j} K_{j k} * P_{i j}(D) w_{i} \\
& =\sum_{|J|=p}^{\prime} \sum_{h} Q_{J}^{k h}(D) L_{J} * \sum_{i} P_{i j_{h}}(D) w_{i} \\
& =\sum_{|J|=p}^{\prime} L_{J} * \sum_{i, h} Q_{J}^{k h}(D) P_{i j h}(D) w_{i} .
\end{aligned}
$$

Now

$$
Q_{J}(D) w_{k}=\sum_{i, h} Q_{J}^{k h}(D) P_{i j_{h}}(D) w_{i}
$$

implies $Q_{J}(D) w_{k}$ has support in $H$ for each $J$ and each $k$. From the above computation we have

$$
u_{k}=\sum_{|J|=p}^{\prime} L_{J} * Q_{J}(D) w_{k}
$$

and therefore

$$
\begin{aligned}
\sum_{k} P_{k l}(D) u_{k} & =\sum_{|J|=p}^{\prime} L_{J} * Q_{J}(D) \sum_{k} P_{k l}(D) w_{k} \\
& =\sum_{|J|=p}^{\prime} Q_{J}(D) L_{J} * \sum_{k} P_{k l}(D) w_{k} \\
& =\sum_{k} P_{k l}(D) w_{k}
\end{aligned}
$$

where the first equality follows from the fact that $Q_{J}(D) w_{k}$ has support in $H$ and the second from the fact that $P^{t}(D) w$ has support in $H$.

Proof of Theorem 2. By the lemma we may assume $p=1$. Thus $P(z)=$ $\left(P_{1}(z), \ldots, P_{q}(z)\right)$. Suppose first that $P_{1}(z), \ldots, P_{q}(z)$ have no common zero in $U+i R^{n}$ where $U$ is a convex open subset of $\Gamma_{0}^{+}$such that $t U \subseteq U$ if $t>1$ and $\Gamma_{0}^{+}$ is the union of $t U$ for $t>0$. Locally in $U+i R^{n}$ we can find holomorphic functions $F_{j}$ such that $\sum P_{j}(z) F_{j}(z)=1$. By Cartan's Theorem B [6, Theorem 7.4.3] these local solutions may be modified to fit together to give global holomorphic functions $F_{j}$ (here we use the convexity of $U$ ). Moreover by [8, Theorem 1] we may choose the holomorphic functions $F_{j}$ so that

$$
\left|F_{j}(z)\right| \leqslant C(1+|z|)^{N} d(\xi)^{-m}, \quad z \in U+i R^{n},
$$

for some constants $C, N$ and $m$. Here $\xi$ is the real part of $z$ and $d(\xi)$ is the minimum of 1 and the distance from $\xi$ to the boundary of $U$. By [10, Proposition 6, p. 306] $F_{j}$ is the Laplace transform of a distribution $K_{j}$. Then $\Sigma P_{j}(D) K_{j}=\delta$ and it remains to locate the support of $K_{j}$. That supp $K_{j}$ is contained in $\Gamma$ follows directly by estimating

$$
\left\langle K_{j}, \phi\right\rangle=(2 \pi)^{-n} \int F_{j}(\xi+i \eta) \hat{\phi}(i \xi-\eta) d \eta
$$

where $\phi \in \mathcal{E}$ has support in a compact convex set disjoint from $\Gamma$ and $\hat{\phi}$ is the Fourier transform of $\phi$. The integral is independent of $\xi \in \Gamma_{0}^{+}$and we simply 
separate $\Gamma$ and supp $\hat{\phi}$ by a hyperplane with normal $\xi \in \Gamma_{0}^{+}$and let $|\xi| \rightarrow \infty$. Alternately supp $K_{j}$ is contained in $\Gamma$ by [10, Remark 1, p. 310].

For the converse we modify the argument in [1, Theorem 3.5]. Assume there exist $K_{j} \in \mathscr{D}_{\Gamma}^{\prime}$ such that $\Sigma P_{j}(D) K_{j}=\delta$. Choose $\phi \in \mathcal{E}$ with $\phi(x)=1$ if $|x|<1$ and $\phi(x)=0$ if $|x|>2$. Then $\Sigma P_{j}(D)\left(\phi K_{j}\right)=\delta+g$ where $g \in \mathscr{D}^{\prime}$ and supp $g \subseteq$ $\{x \in \Gamma|1<| x \mid \leqslant 2\}$. Let $G_{j}$ be the Laplace transform of $\phi K_{j}$ and let $G$ be the Laplace transform of $g$. Then $G$ and the $G_{j}$ are entire functions and $\sum P_{j}(z) G_{j}(z)=$ $1+G(z)$. By the Paley-Wiener theorem [2, p. 211]

$$
|G(z)| \leqslant C(1+|z|)^{N} e^{h(-\xi)}
$$

where $z=\xi+i \eta$ and where $h(-\xi)=\sup \{-\langle\xi, x\rangle|x \in \Gamma, 1 \leqslant| x \mid<2\}$. If $\xi \in \Gamma_{0}^{+}$ then $\langle\xi, x\rangle>0$ for each $x \in \Gamma, x \neq 0$ and so $h(-\xi)=-\operatorname{dist}\left(\xi, \partial \Gamma_{0}^{+}\right)$. Here $\operatorname{dist}\left(\xi, \partial \Gamma_{0}^{+}\right)=\inf \{\langle\xi, x\rangle|x \in \Gamma| x \mid,=1\}$ is easily seen to be the distance from $\xi$ to the boundary of $\Gamma_{0}^{+}$. At any common zero of the $P_{j}$ we have $G(z)=-1$. Thus for some constants $C$ and $N$ we have

$$
\operatorname{dist}\left(\xi, \partial \Gamma_{0}^{+}\right) \leqslant C+N \log (1+|z|)
$$

if $\xi \in \Gamma_{0}^{+}, z=\xi+i \eta$ and $P_{j}(z)=0, j=1, \ldots, q$.

Suppose now $\Gamma$ is semialgebraic. First note $\Gamma_{0}^{+}$is the complement of the projection on the first $n$ coordinates of the set of $(\xi, x)$ such that $\xi \in R^{n}, x \in \Gamma$, $x \neq 0,\langle\xi, x\rangle<0$ and hence is semialgebraic by the Seidenberg-Tarski theorem. It follows that the set of $(\mu, \xi, x)$ such that $\left.\xi \in \Gamma_{0}^{+}, x \in \Gamma,|x|=1, \mu\right\rangle\langle\xi, x\rangle$ is semialgebraic and hence by the Seidenberg-Tarski theorem the set $M$ of $(\mu, \xi)$ such that $\xi \in \Gamma_{0}^{+}$and $\mu>\operatorname{dist}\left(\xi, \partial \Gamma^{+}\right)$is semialgebraic. An application of the Seidenberg-Tarski theorem shows that the closure and interior of a semialgebraic set is semialgebraic. Thus $\partial M \cap\left(R \times \Gamma_{0}^{+}\right)=\left\{(\mu, \xi) \mid \xi \in \Gamma_{0}^{+}, \mu=\operatorname{dist}\left(\xi, \partial \Gamma^{+}\right)\right\}$is semialgebraic. This property of the distance function, that the graph is semialgebraic, is known in other cases as well but is particularly simple to prove in our case because we have a nice formula for the distance to the boundary of a convex cone. It now follows that the set $L_{0}$ of $(\mu, \tau, \xi, \eta)$ such that $\xi \in \Gamma_{0}^{+}, \mu=\operatorname{dist}\left(\xi, \partial \Gamma^{+}\right), \tau \geqslant \mid \xi+$ $i \eta \mid, P_{j}(\xi+i \eta)=0, j=1, \ldots, q$, is semialgebraic. Again by the Seidenberg-Tarski theorem the projection $L$ on the first two coordinates is semialgebraic. By (4) if $(\mu, \tau) \in L$ then $\mu \leqslant C+N \log (1+\tau)$. By [5, Lemma 2.1, p. 276] it follows that there is a constant $C_{1}$ such that $\mu \leqslant C_{1}$ if $(\mu, \tau) \in L$. Now let $U=\{\xi \in$ $\left.\Gamma_{0}^{+} \mid \operatorname{dist}\left(\xi, \partial \Gamma^{+}\right)>C_{1}\right\}$.

APPENDIX. We now show $\delta_{\Gamma}^{\prime}$ is a subring of $\mathscr{D}_{\Gamma}^{\prime}$. Suppose $f, g \in \mathcal{S}_{\Gamma}^{\prime}$. Then

$$
e^{-\langle\xi, \cdot\rangle}(f * g)=\left(e^{-\langle\xi,\rangle} f\right) *\left(e^{-\langle\xi,\rangle} g\right) \text {. }
$$

For each $\xi \in \Gamma_{0}^{+}$the factors on the right are in $\mathcal{S}^{\prime}$ and therefore by [10, Corollary, p. 302] are in $\theta_{C}^{\prime}$. It follows that $e^{-\langle\xi,\rangle}(f * g)$ is in $\theta_{C}^{\prime}$. Taking Fourier transforms we obtain

$$
\left(e^{-\langle\xi, \cdot\rangle}(f * g)\right)^{\wedge}(\eta)=F(\xi+i \eta) G(\xi+i \eta)
$$

where $F$ (respectively $G$ ) is the Fourier transform of $f$ (respectively $g$ ). The product on the right is a holomorphic function in $\Gamma_{0}^{+}+i R^{n}$ and by [7, Theorem 1] is the Laplace transform of a distribution $u \in \mathcal{S}_{\Gamma}^{\prime}$. Obviously $u=f * g$. 


\section{REFERENCES}

1. M. F. Atiyah, R. Bott and L. Garding, Lacunas for hyperbolic differential operators with constant coefficients, Acta Math. 124 (1970), 109-189.

2. W. F. Donoghue, Jr., Distributions and Fourier transforms, Academic Press, New York, 1969.

3. A. Enqvist, On fundamental solutions supported by a convex cone, Ark. Mat. 12 (1974), 1-40.

4. $ـ$ On temperate fundamental solutions supported by a convex cone, Ark. Mat. 14 (1976), $35-41$.

5. L. Hörmander, Linear partial differential operators, Springer-Verlag, Berlin, 1963.

6. __ An introduction to complex analysis in several variables, Van Nostrand, Princeton, N.J., 1966.

7. B. E. Petersen, On the Laplace transform of a temperate distribution supported by a cone, Proc. Amer. Math. Soc. 35 (1972), 123-128.

8. Holomorphic functions with growth conditions, Trans. Amer. Math. Soc. 206 (1975), $395-406$.

9. Holomorphic functions with bounds, Complex Analysis and its Applications, Vol. III, International Atomic Energy Agency, Vienna, 1976, pp. 107-120.

10. L. Schwartz, Théorie des distributions, new ed., Hermann, Paris, 1966.

11. K. T. Smith, Formulas to represent functions by their derivatives, Math. Ann. 188 (1970), 53-77.

Department of Mathematics, Oregon State University, Corvallis, Oregon 97331 